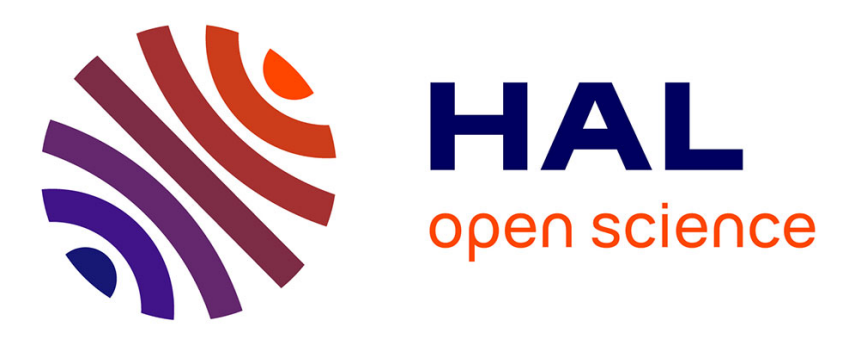

\title{
An introduction to photocatalysis through methylene blue photodegradation
}

Matthieu Petit, Lisa Michez, Jean-Manuel Raimundo, Tuhiti Malinowski, Philippe Dumas

\section{- To cite this version:}

Matthieu Petit, Lisa Michez, Jean-Manuel Raimundo, Tuhiti Malinowski, Philippe Dumas. An introduction to photocatalysis through methylene blue photodegradation. European Journal of Physics, 2016, 37 (6), pp.065808. 10.1088/0143-0807/37/6/065808 . hal-01382652

\section{HAL Id: hal-01382652 \\ https://hal-amu.archives-ouvertes.fr/hal-01382652}

Submitted on 17 Oct 2016

HAL is a multi-disciplinary open access archive for the deposit and dissemination of scientific research documents, whether they are published or not. The documents may come from teaching and research institutions in France or abroad, or from public or private research centers.
L'archive ouverte pluridisciplinaire HAL, est destinée au dépôt et à la diffusion de documents scientifiques de niveau recherche, publiés ou non, émanant des établissements d'enseignement et de recherche français ou étrangers, des laboratoires publics ou privés. 


\title{
An introduction to photocatalysis through methylene blue photodegradation
}

\author{
Matthieu Petit, Lisa Michez, Jean-Manuel Raimundo, Tuhiti \\ Malinowski, Philippe Dumas \\ Aix-Marseille Université, CNRS, CINaM UMR 7325, 13288, Marseille, France. \\ E-mail: matthieu.petit@univ-amu.fr
}

\begin{abstract}
We described a simple experimental set-up for a lab work on the photocatalytic degradation of methylene blue by $\mathrm{TiO}_{2}$ nanoparticles. The photocatalysis process can be used for many applications. Treatments for diluted wastewaters industries, air purifying in underground car parks, preventing fouling on glass surfaces, these are some of the things this phenomenon has the ability to do. The described experiment is easy to perform and the interpretation can be easily adapted to different level of students: from high school students demonstrating their interests on a sustainable development, to students who are getting Masters in the science department that want to propose a full explanation of all the phenomenon of the photocatalytic process. Starting by a description of the experimental set-up, we analysed the photocatalyst nanoparticles and applied the Langmuir-Hinshelwood model to our experimental data. Finally we discussed shortly on the respective energetic levels of the photocatalyst semiconductor and the methylene blue.
\end{abstract}

PACS numbers: 82.45.Jn, 82.50.-m, 82.65.+r, 77.84.Cg

Keywords: photocatalysis, titanium dioxide, $\mathrm{TiO}_{2}$, Langmuir-Hinshelwood, methylene blue, degradation, redox reaction, dyes

\section{Introduction}

\subsection{Context}

The last IPCC (Intergovernemental Panel on Climate Change) synthesis report has confirmed the impact of mankind on the climate change and its impacts on the ecosystems, people and economics. However this report also emphasizes that there are several means to limit climate change and its consequences [1]. The efficient use of solar energy, as a green energy source for various applications is one of these means that the scientific and industrial communities tend to develop: the solar power irradiating the surface of Earth (around $10^{5} \mathrm{TW}$ ) is four orders of magnitude higher than the current 
global human energy consumption. So the photocatalysis belongs to this wide family of various techniques and technologies aiming at a sustainable development of our society. Reports on photocatalytic reactions date from the beginning of the 20th century: for example C. F. Goodeve and J. A. Kitchener described the "photosensitisation of solids" and the bleaching of dyes by $\mathrm{TiO}_{2}$ in 1938 [2]. The true boost of interest on photocatalysis occurred in 1972 with the re-publication in English of a previous report of the splitting of water in hydrogen and oxygen at the surface of $\mathrm{TiO}_{2}$ under ultraviolet irradiation by A. Fujishima and K. Honda [3]. Nowadays advances in photocatalysis science and technology, as well as in industrial applications, point out the promising potential of photocatalysis for reducing the levels of greenhouse gases in the atmosphere, for green chemistry, degrading water pollutants, etc.

Regarding students with majors in physics or chemistry, the main interest of photocatalysis lies in the understanding of the process and the ability to draw links between these fields: photo- and electrochemistry, material and surface sciences, solid physics and so on. Photocatalysis is actually an interdisciplinary domain.

\subsection{Definition of some terms}

So as to describe the photocatalytic process, we will use in this article some concepts that belong usually to the vocabulary of chemistry. We provide here simple definitions of these concepts:

- activation energy: it is the minimum energy needed to start a chemical reaction which transforms reactants to products. Therefore the activation energy is a potential barrier separating two energetic states formed by the reactants (starting state) and the products (final state).

- adsorption, physisorption, chemisorption and desorption: we would like to emphasize the distinction between the notions of adsorption of atoms or molecules on a surface, which may include physisorption (weak bonding, typically Van der Walls forces) and chemisorption (covalent bonding between the adsorbed molecules and the surface). The desorption of atoms or molecules from a surface is the release of these species from the surface (the previous bondings are broken). We wish to highlight the difference between the adsorption of molecules on a surface and the light absorption (photoabsorption) by the semiconductor material to potentially create electron/hole pairs. Several models can be used to describe the adsorption phenomena, like the Langmuir isotherm. These models basically give a relationship between the amount of species in a gas or liquid and the amount of these species adsorbed on a surface at a given temperature.

- reduction-oxidation (redox reactions): redox reactions are a family of chemical reactions involving an exchange of charges (electrons) between atoms or molecules. If an exchange of charges takes place, it means that a potential difference exists between the various chemical species. Every chemical species has its own potential called the redox potential (in volt). It provides a measure of the ability of a 
species to give up (and thus be oxidized) or to acquire an electron (and thus be reduced). The redox potential is an intrinsic parameter of a given species. So as to be compared and forecast which chemical species will give or accept electrons from another species, the redox potential are organized on a scale. The origin of the scale is arbitrarily attributed to the hydrogen species $\left(\mathrm{H}_{2} / \mathrm{H}^{+}\right)$: the so-called standard hydrogen electrode (SHE) whose the redox potential is thus equal to zero.

- vacuum level: this is a notion of solid state physics. the vacuum level is the energy of an electron in a perfect vacuum meaning infinitely far away from any materials. This level is used as an absolute origin for the energy (or potential) of electrons.

- chemical or reaction kinetics: when a chemical reaction occurs, reactants " $A$ " are transformed into products " $B$ ". Such a transformation takes time. Reaction kinetics is the study of the rate " $r$ " of the chemical reactions. $r$ depends on the concentration of $A$ and $B$ and on $k$ the reaction rate constant:

$$
r=k[A]^{x}[B]^{y}
$$

$x$ and $y$ are called the partial orders of the reaction (and are integers). For example, if the reaction is of first order, $x+y=1$. It means that the rate depends only of the concentration of one reactant:

$$
r=-\frac{d[A]}{d t}=k[A]
$$

We will use this case to describe the photocatalytic degradation of methylene blue.

\subsection{Definition of photocatalysis}

Photocatalysis comes from two words: light and catalysis. Various definitions of "photocatalysis" have been suggested. One the most commonly used definitions refers to a chemical reaction induced by the absorption of light (photo-absorption) by a solid material (generally a semiconductor). The reaction occurs at the surface of the semiconductor which remains unchanged during and after the reaction. This semiconductor is called a photocatalyst. From a thermodynamically point of view, the catalysis and the photocatalysis are two different processes. A "simple" catalyst will enhance the reaction rate of an already thermodynamically allowed chemical reaction by reducing the activation energy. On the contrary, thanks to the energy brought by light, photocatalysis can promote reactions unfavourable on a thermodynamic point of view. This is the example of the photocatalytic splitting of water into hydrogen and oxygen. From this point of view catalysis and photocatalysis concepts are basically different $[4,5]$. 


\section{Brief theoretical description}

\subsection{Overall steps}

Photocatalysis can occur in liquid or gas phase. The whole photocatalysis process can be divided into five independent steps that are common to the heterogeneous catalysis as clearly described by J.M. Hermann [6]:

(i) Diffusion of the reactants in the fluid phase to the surface of the photocatalyst.

(ii) Adsorption of reactants on the surface of the photocatalyst: classical models like Freundlich or Langmuir isotherms can describe this step.

(iii) Reaction in the adsorbed phase. It is often basically a charge transfer between adsorbed reactants and the semiconductor: a so-called redox reaction. Light is absorbed by the semiconductor: electrons are "available" in the conduction band for the redox reaction.

(iv) Desorption of the products of the redox reaction (i.e release of the products from the surface of the photocatalyst).

(v) Draining of the products from the region close to the semiconductor to free the access to its surface for the remaining reactants.

The third step is the heart of the photocatalytic process. This is when the transformation of chemical species like degradation of pollutants takes place.

\subsection{Interface between the semiconductors and the fluid phase}

To explain and describe the redox reaction occurring at this step, we need to bring together the redox potential scale used to know the potential of the chemical reactants in the liquid or gas phase and the energy levels structure of the semiconductor. In this way, we must link electrochemistry and solid physics. This is done by analysing the properties of the liquid(gas)/solid interface. Such an interface can be handle in the same way as a p-n junction (chapter 4, ref.[7]) with an energetic description of the electrolyte following the model developed by H. Gerischer [8, 9]. Therefore the relationship between the two energy scales is the following equation (eq.3) $[10,11,12]$ :

$$
E(e V)=-q \mathcal{E}(V)-\Phi
$$

$\mathrm{E}$ is the value of the energy relative to the vacuum level, $\mathcal{E}$ is the redox potential referenced to the SHE, $\mathrm{q}$ is the elementary charge and $\Phi$ is the absolute electrode potential of the redox couple $\left(H_{2} / H^{+}\right)$on which is based the SHE. It means that $\Phi$ is the value of the SHE potential compared to the vacuum level. Its value is experimentally determined and is equal to about $4.5 \mathrm{eV}$. Thanks to this common scale between redox couples and semiconductors energy levels it is possible to know if a redox reaction is favourable between a given semiconductors and reactants. 


\subsection{Langmuir-Hinshelwood (LH) model}

The LH model is often used to quantify the photocatalytic process even if there are some discussions over its relevance and the use of it according to a given photocatalytic process. It is not the purpose of this article to present these discussions but we recommend the reader to read the paper of B. Othani [4].

During the photocatalytic process there is a transformation of the reactants adsorbed onto the surface of the semiconductor, the concentrations of these chemical species vary, hence these processes are kinetic reactions. The LH model takes into account the phenomenon relative to the adsorption on the semiconductor surface and the kinetic reactions [13]. The main equation of the $\mathrm{LH}$ model is eq.4:

$$
\frac{1}{v_{L H}}=\frac{1}{k}+\frac{1}{k K} \times \frac{1}{C_{i}}
$$

where $v_{L H}$ is the rate of the reaction (the photocatalytic rate), $\mathrm{k}$ is the rate constant of the reaction of the adsorbed reactants on the semiconductors surface with $e^{-}$or $h^{+}$created by photoabsorption, $\mathrm{K}$ is the adsorption equilibrium constant (Langmuir isotherms) and $C_{i}$ is the initial concentration of reactants before the beginning of the photocatalysis. Then it is easy to check the validity of the LH model to describe a photocatalytic process by drawing $\frac{1}{v_{L H}}$ versus $\frac{1}{C_{i}}$ and obtaining a straight line.

\section{Method and implementation}

\subsection{Equipment}

To provide evidences of the photocatalytic effect in the framework of our practical course, we use methylene blue (MB) (CAS number 7220-79-3) as the organic molecules to degrade. MB is non-toxic hence it is suitable for an educational lab work. Methylene blue absorbs light in the visible range, therefore the measurements of its concentration can easily be determined using the Beer-Lambert law (eq.5):

$$
A(\lambda)=\log \left(\frac{I(\lambda)}{I_{0}(\lambda)}\right)=-\epsilon l[B M]
$$

A is the absorbance at a given wavelength $\lambda, I_{0}(\lambda)$ represents the incident light intensity, $I(\lambda)$ the light intensity transmitted trough the MB solution, $\epsilon$ is the molar attenuation coefficient of MB (the coefficient of light absorption by a liquid solution of $\mathrm{MB}), 1$ the path length of the beam of light through the material sample and finally [MB] the concentration of methylene blue in the solution. To measure the light spectrum and intensity, we use an educational version of the USB-650 Red-Tide spectrophotometer from Ocean Optics [14]. Titanium dioxide ( $\mathrm{TiO}_{2}$, CAS number 13463-67-7) is used as the semiconductor acting as the photocatalyst. We used $\mathrm{TiO}_{2}$ nanopowder with an average size of $21 \mathrm{~nm}$ because according to ref [15] the photocatalytic degradation increases especially for particles with sizes less than $30 \mathrm{~nm}$. $\mathrm{TiO}_{2}$ has a bandgap around 3-3.2 eV according to its phase (anatase or rutile). So the suitable wavelength light for 
the excitation of $\mathrm{TiO}_{2}$ is in the UV range of the electromagnetic spectrum. $\mathrm{TiO}_{2}$ is one of the most commonly used photocatalyst because of its chemical stability, non-toxicity, price and even if its bandgap lies in the UV part of the visible spectra which count for only $5 \%$ of the sunlight. The UV excitation light is a commercial UV energy saving lamp with a power of $20 \mathrm{~W}$. The spectrum of the lamp is shown on fig. $1 \mathrm{~b}$ ).

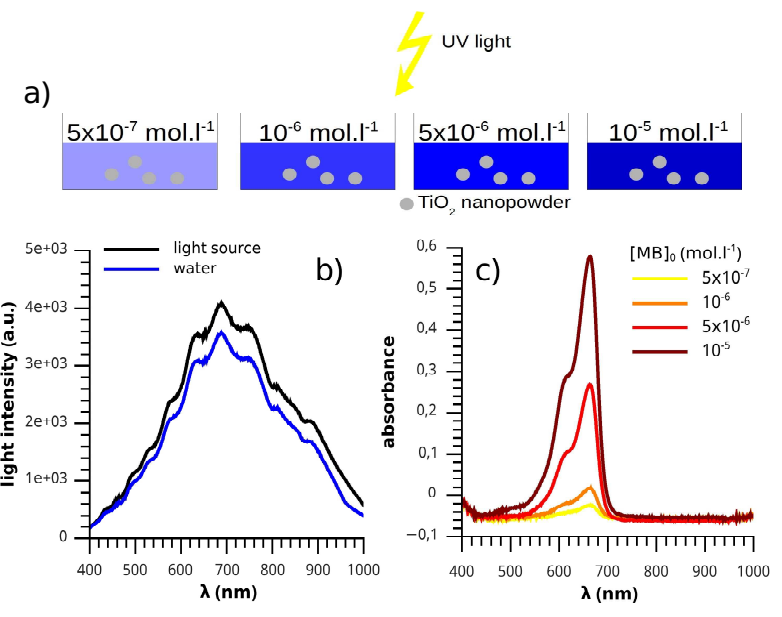

Figure 1. (colour online) a) Scheme of the experimental set-up. b) UV-VIS spectrum of the light source and the solvent (water). c) Absorbance spectra of the four initial MB concentrations.

\section{2. method}

3.2.1. $M B$ solutions: Students prepare four $\mathrm{MB}$ solutions of different initial concentrations $[M B]_{0}$ in the range of $5 \times 10^{-7}$ to $10^{-5}$ mol.l $l^{-1}$ with water as the solvent (fig.1 a)). This range is determined according to the sensing capability of the spectrophotometer and on the other hand the concentration validity domain of the Beer-Lambert law. Then they set up four crystallisers with $10 \mathrm{mg}$ of $\mathrm{TiO}_{2}$ nanopowder in each and $50 \mathrm{ml}$ of each initial concentration. The powder should be well mixed in water in order to avoid the aggregation of particles which can entail a decrease of the specific surface of the $\mathrm{TiO}_{2}$ photocatalyst. A set of four control experiments (same experimental parameters but without $\mathrm{TiO}_{2}$ particles) should also be prepared to ascertain the photocatalytic effect and disambiguate with other phenomena such as the photolysis of MB molecules. The photolysis is the degradation of the molecules directly by the sole effect of light. The Fig.1 b) displays the spectrum of water as the reference for the measurement of the MB concentration via the absorbance. Then Fig.1 c) presents the absorbance spectra of the four initial MB solutions with chosen concentrations of $5 \times 10^{-7}, 10^{-6}, 5 \times 10^{-6}$ and $10^{-5}$ mol. $l^{-1}$. The main absorption peak of MB is at 665 $\mathrm{nm}$. Students should use this wavelength throughout the practical work to conduct the monitoring of the evolution of the absorbance versus time of irradiation to maximise the accuracy of the measures. 
3.2.2. measurements process: At an irradiation time $\mathrm{t}=0$, the students put the four crystallisers under UV light and they follow the evolution of the concentration of $\mathrm{MB}$ versus the irradiation time. They choose a time interval $t_{i}$ (10 min for instance) and every $t_{i}$ they sample a small volume $(2-3 \mathrm{ml})$ of the MB solutions ideally with a syringe. They performe the absorbance measurements on these volumes so as to follow the evolution of the MB concentrations versus time of irradiation. To increase the accuracy of the absorbance values, the sampled volumes of $\mathrm{MB}$ solutions should be centrifuged in order to remove $\mathrm{TiO}_{2}$ particles from the solutions which might disrupt the spectroscopic measurements.

\section{Results and discussion}

\section{1. $\mathrm{TiO}_{2}$ characterisation, quantum size effect}

The photocatalyst plays a key role in the photocatalytic process since it provides charges needed for the redox reactions by the absorption of light. Therefore the yield of the whole photocatalytic process is linked to the bandgap of the semiconductor. That is why we encourage the students to try to characterise the used semiconductor. X-ray diffraction (XRD) can be easily performed on the $\mathrm{TiO}_{2}$ nanopowder. Fig.2 shows the XRD diagram of the nanopowder recorded on a Philips PANanalytical XPert XRD system with a $\mathrm{Cu}$ electrode. The X-ray source is non monochromatic. The peaks identification shows that the powder is a mix of anatase and rutile which are two well-known forms of titanium dioxide. From the quantitative analysis of the XRD spectrum, this allows us to determine that there are $83 \%$ of anatase and $17 \%$ of rutile in the powder.

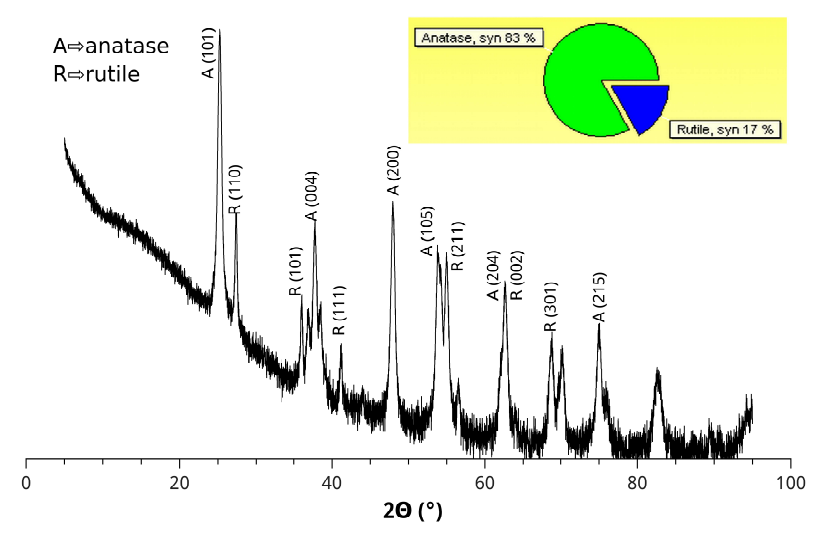

Figure 2. XRD diagram of the $\mathrm{TiO}_{2}$ nanopowder used as a photocatalyst.

Then the size of the nanoparticles of $\mathrm{TiO}_{2}$ can be estimated from the XRD diagram by using the Scherrer equation (eq. 6). It links the size of the particles to the width of the XRD peaks [16]:

$$
\tau=\frac{k \lambda}{\sqrt{H^{2}-s^{2}} \cos (\theta)}
$$


Table 1. Values of parameters used to predict the shift of the energy bandgap due to quantum size effect in the $\mathrm{TiO}_{2}$ nanoparticles (anatase)

\begin{tabular}{ll}
\hline for $\mathrm{TiO}_{2}$ & Values \\
\hline $\mathrm{r}$ & $10 \mathrm{~nm}$ \\
$\frac{m_{e}^{*}}{m_{0}}$ & $0.095[19]$ \\
$\frac{m_{h}}{m_{0}}$ & $0.200[19]$ \\
$\frac{\epsilon}{\epsilon_{0}}$ & 90 \\
\hline
\end{tabular}

$\tau$ is the mean size of a grain size (to be precise this the size of the crystalline domains which can be smaller or equal to a grain size), $\mathrm{k}$ is a dimensionless factor with a value around $0.9, \lambda$ is the X-ray wavelength (in our case the main wavelength is $1.5406 \AA$ ), $\mathrm{H}$ is the line broadening at half the maximum intensity (FWHM), $\mathrm{s}$ is the instrumental line broadening (evaluated to $0.03^{\circ}$ for our apparatus) and $\theta$ is the Bragg angle. All angles should be in radians.

Using the XRD peak indexed A(101) on Fig.2 at a position of $25.24^{\circ}$, the estimated average size of the $\mathrm{TiO}_{2}$ nanoparticles is found to be $20.2 \mathrm{~nm}$. The supplier provides a value of $21 \mathrm{~nm}$, therefore this is very pertinent (product ref 718467 Aldrich [17]).

Because the particles of $\mathrm{TiO}_{2}$ are nanometer-sized, students with a background in nanoscience may think about discussing a possible quantum size effect on the bandgap of these nanoparticles. Changing the bandgap energy will change the absorbed light and impact of the photocatalytic degradation rate through a possible variation of a number of electron/hole pairs created. Quantum size effects generally occur for semiconductor particles of a size in the range of 10 to $100 \AA$ due to quantum confinement. It might entail an energy shift $\Delta E$ of the bandgap which can be calculated with the following approximated equation (eq.7) [18]:

$$
\Delta E=\frac{\hbar^{2} \pi^{2}}{2 r^{2}}\left(\frac{1}{m_{e}^{*}}+\frac{1}{m_{h}^{*}}\right)-\frac{1.786 q^{2}}{\epsilon r}-0.248 \frac{q^{4}}{2 \epsilon^{2} h^{2}} \frac{m_{e}^{*}+m_{h}^{*}}{m_{e}^{*} m_{h}^{*}}
$$

$\mathrm{r}$ is the nanoparticle radius, $\mathrm{q}$ is the elementary charge of the electron, $m_{e}^{*}, m_{h}^{*}$ are the effective masses of electrons and holes, $\epsilon$ is the dielectric constant and $h$ the Planck's constant. This equation is rather complex but the purpose here is simply to get a relationship between the small size of the particles and a possible energetic effect on the bandgap without going into solid physics details.

Using the values of the parameters for $\mathrm{TiO}_{2}$ listed in table $1, \Delta E$ is evaluated to $0.02 \mathrm{eV} . \Delta E$ is insignificant in our context since the corresponding change in absorbed light wavelength will be around $2 \mathrm{~nm}$.

\section{2. $M B$ concentration versus time of $U V$ irradiation}

The evolution of the MB concentration for the four prepared solutions is presented in fig. 3 on a logarithmic scale. The accuracy of the concentrations measurements is 
Table 2. Results coming from the fits of fig. 3 and calculated values for the LH model

\begin{tabular}{lll}
\hline$[M B]_{0}$ mol.l $l^{-1}$ & $k_{r}(\mathrm{eV})$ & $\frac{1}{v_{L H}}$ \\
\hline $5 \times 10^{-7}$ & 0.0199 & $8.69 \times 10^{7}$ \\
$10^{-6}$ & 0.0319 & $3.13 \times 10^{7}$ \\
$5 \times 10^{-6}$ & 0.0157 & $1.18 \times 10^{7}$ \\
$10^{-5}$ & 0.0094 & $1.06 \times 10^{7}$ \\
\hline
\end{tabular}

subdued at low concentrations because of the limitation of the sensitivity of our UVVIS spectrophotometer for very low absorbances. From the linear relationship between $\ln ([\mathrm{MB}])$ and $t$ observed on fig.3, the reaction rate of MB degradation is of apparent first order. On this point readers should refer to the comment made by Herrman in [13], section 2.5 regarding the apparent contradiction on the increase of the reaction rate with the decrease of the initial concentration. Avoiding the confusion between the reaction rate and the conversion rate solves this contradiction. The first one depends on the initial concentration wherea the second one does not.

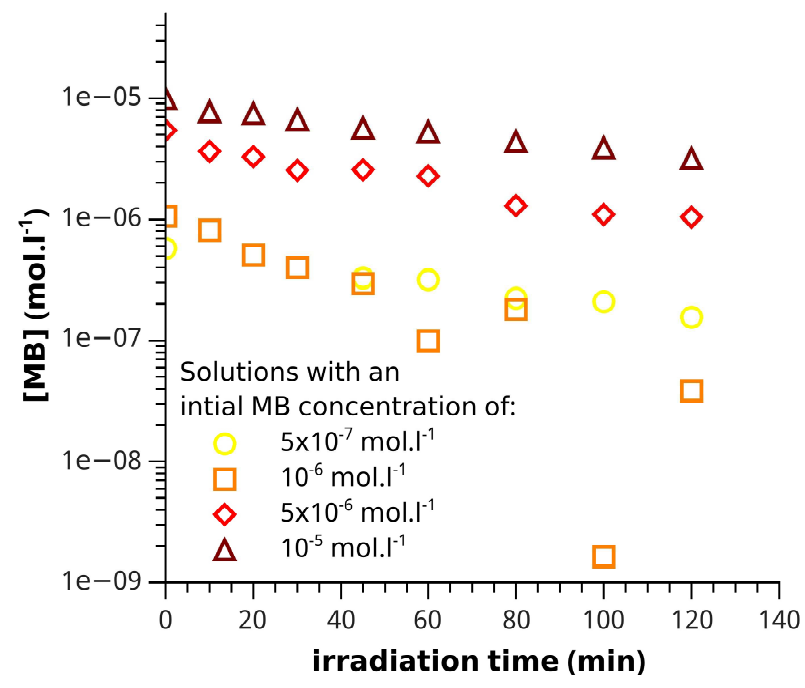

Figure 3. Evolution of the methylene blue concentration [MB] versus the irradiation time for the four initial solutions.

Using a first order reaction, the integration of the reaction rate gives (eq. 8):

$$
\frac{-d[M B]}{d t}=k_{r}[M B] \Rightarrow \ln ([M B])=\ln \left([M B]_{0}\right)-k_{r} t
$$

By fitting the experimental data of fig.3, students can extract the values of the kinetic constants $k_{r}$ for the four experiments. The fitted values are given in table 2 .

\subsection{LH model}

Moreover the photocatalytic degradation rate $v_{L H}$ is estimated from $k_{r}$ and the LH model can be verified by plotting $1 / v_{L H}$ versus $1 /[M B]_{0}$ (fig.4). 


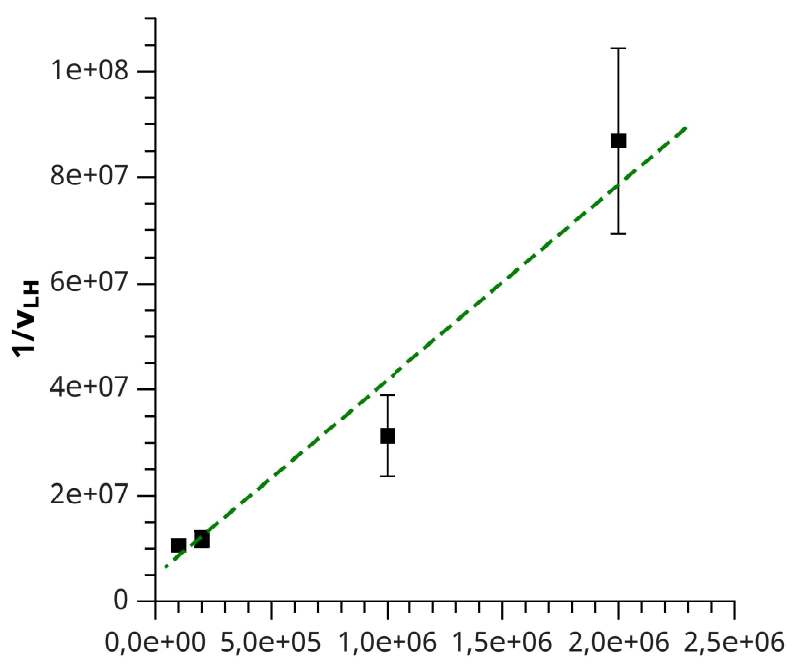

$1 /[\mathrm{MB}]_{0}$

Figure 4. Verification of the LH model.

The linear relationship between $1 / v_{L H}$ and $1 /[M B]_{0}$ is fully in agreement with eq.4. The correlation factor of the linear fitting is equal to 0.96. It is a bit low but the impact of the last experimental point at the lowest MB concentration is significant. As discussed above, it comes from the limitation of our UV-VIS spectrophotometer in the low range concentration of $\mathrm{MB}$ solutions. The value of $\mathrm{K}$ obtained by the linear fitting is $5.9 \times 10^{4}$ l. $\mathrm{mol}^{-1}$ and it is typical of the degradation of organic molecules soluble in water.

\section{4. $M B$ degradation pathway}

The photocatalytic degradation of MB does not result from a direct redox reaction between the $\mathrm{TiO}_{2}$ nanoparticles and the MB molecules. The photocatalytic degradation pathway of MB in water has been proposed by Houas \& al [20]. The electrons generated in the $\mathrm{TiO}_{2}$ by the UV light either recombine with the holes of the valence band or react with the adsorbed oxygen on $\mathrm{TiO}_{2}$ surface. In the latter case, oxygen ions are created and react in turn with the adsorbed water molecules. Hydroxyl radicals $\mathrm{OH}^{\circ}$ and hydroxyl groups are formed. Eventually the MB molecules are degraded by the reactions with the radicals. The establishment of the whole degradation pathway by the students is not the goal of this practical work. However students can comment on the feasibility of the degradation of $\mathrm{MB}$ by $\mathrm{TiO}_{2}$ by drawing the energetic diagram with the different levels positions of anatase, rutile and MB by using eq.3 (fig.5).

From this diagram it can be concluded that the direct charge transfers between the different phases of $\mathrm{TiO}_{2}$ and $\mathrm{MB}$ molecules are unlikely. Therefore the photocatalytic degradation of $\mathrm{MB}$ by $\mathrm{TiO}_{2}$ will need intermediate steps involving $\mathrm{OH}^{\circ}$ radicals to be processed. 


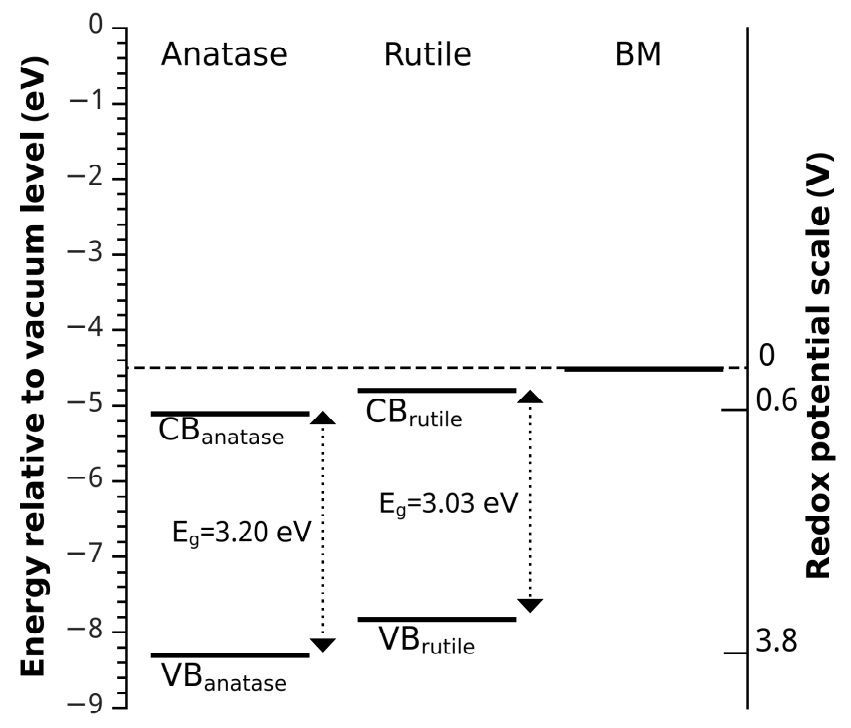

Figure 5. Energy diagram with the positions of the MB redox potential, $\mathrm{CB}$ and $\mathrm{VB}$ of anatase and rutile [21].

\section{Conclusion}

In this paper we described a practical work consisting in the photodegradation of $\mathrm{MB}$ by $\mathrm{TiO}_{2}$ nanoparticles. This lab work only requires a simple experimental set-up which allows us to provide evidences of the significance of the photocatalysis. However, this phenomenon is rather complex and requires several notions from chemistry and physics. A full and informational explanation of the phenomenon must be made, and the report must include the following : diffusion, adsorption, redox reactions, excitation of semiconductors by light absorption and other aspects of the photocatalysis process. We have thus discussed the properties of the photocatalyst in terms of materials science using XRD diffraction and considering a potential quantum size effect on the $\mathrm{TiO}_{2}$ bandgap because of the size of the nanoparticles. Then we checked the validity of the main model developed to describe the photocatalysis effects, the Langmuir-Hinshelwood model and eventually we commented on the photodegradation pathway of MB by analysing the respective energetic levels positions of the photocatalyst and the organic molecules. This analyse indicate that a direct charge transfer between $\mathrm{TiO}_{2}$ and $\mathrm{MB}$ molecules is unlikely so the photodegradation pathway may use intermediate steps like formation of hydroxyl radicals. It is a lab work that allows students to draw links between several different science domains that have been historically explored at different places and times.

To go further in the photocatalytic processes, students can look at the different means developed to enhance the photocatalysis rate such as the improvements of the semiconductor photoelectrode. As an example involving nanoscience, we can cite the socalled plasmonic photocatalysis where metal nanoparticles added to the semiconductors improved the photocatalytic performance through localised surface plasmon resonance 
$[22]$.

\section{References}

[1] Team Core Writing 2015 IPCC, 2014: Climate Change 2014: Synthesis Report. Contribution of Working Groups I, II and III to the Fifth Assessment Report of the Intergovernmental Panel on Climate Change URL http://ar5-syr.ipcc.ch/ipcc/ipcc/resources/pdf/IPCC_SynthesisReport.pdf

[2] Goodeve C F and Kitchener J A 1938 Transactions of the Faraday Society 34 902-908

[3] Honda A and Fujishima K 1972 Nature 238 37-38

[4] Ohtani B 2010 "Journal of Photochemistry \& Photobiology, C: Photochemistry Reviews" 11 157178 ISSN 1389-5567

[5] Fujishima A, Zhang X and Tryk D A 2008 Surface Science Reports 63 515-582

[6] Herrmann J $\quad$ M 1999 Catalysis Today $\mathbf{5 3}$ 115-129 ISSN 09205861 URL http://linkinghub.elsevier.com/retrieve/pii/S0920586199001078

[7] Kaneko M and Okura I 2002 Photocatalysis: Science and Technology springer-v ed (Berlin Heidelberg New York: Kodansha, Springer) ISBN 3-540-43473-9

[8] Gerischer H 1966 Journal of the Electrochemical Society 113 1174-1182

[9] Smandek B, Chmiel G and Gerischer H 1989 Berichte der Bunsengesellschaft für physikalische Chemie 93 1094-1103

[10] Lohmann F 1967 Zeitschrift für Naturforschung A 22a 843-844 URL http://zfn.mpdl.mpg.de/data/Reihe_A/22/ZNA-1967-22a-0843_n.pdf

[11] Reiss H 1985 Journal of Physical Chemistry 89 3783-3791

[12] Reiss H and Heller A 1985 Journal of Physical Chemistry 89 4207-4213

[13] Herrmann J M 2010 Journal of Photochemistry and Photobiology A: Chemistry 216 85-93 ISSN 10106030 URL http://linkinghub.elsevier.com/retrieve/pii/S1010603010001796

[14] Ocean Optics I [Online; accessed 04-November-2015] URL http://oceanoptics.com/

[15] Xu N, Zaifeng Shi Y F and unhang Dong Jun Shi M Z C H 1999 Industrial \& Engineering Chemistry Research 38 373-379

[16] Patterson A L 1939 Physical Review B 56 978-982

[17] Aldrich S [Online; accessed 17-November-2015] URL http://www.sigmaaldrich.com

[18] Linsebigler A, Lu G and Yates J 1995 Chemical Reviews 95 735-758 ISSN 0009-2665

[19] Zhang J, Zhou P, Liu J and Yu J 2014 Phys. Chem. Chem. Phys. 16(38) 20382-20386

[20] Houas A, Lachheb H, Ksibi M, Elaloui E, Guillard C and Herrmann J m 2001 Applied Catalysis B: Environmental 31 145-157

[21] Scanlon D O, Dunnill C W, Buckeridge J, Shevlin S A, Logsdail A J, Woodley S M, Catlow C R A, Powell M J, Palgrave R G, Parkin I P, Watson G W, Keal T W, Sherwood P, Walsh A and Sokol A A 2013 Nature Materials 12 798-801 ISSN 1476-1122

[22] Zhang X, Chen Y L, Liu R S and Tsai D P 2013 Reports on progress in physics. Physical Society (Great Britain) $\mathbf{7 6} 046401$ ISSN 1361-6633 